\title{
Actuar-Enseñar entre la Diversidad: Construyendo Educación Inclusiva en Atacama
}

\author{
Act-Teach among Diversity: Building Inclusive Education in \\ Atacama
}

\author{
Adriana Fernández Muñoz * \\ Daniela Durán Rojas \\ Universidad de Atacama, Chile
}

\begin{abstract}
La educación chilena ha transitado en búsqueda de calidad e inclusión de todos y todas, respondiendo a las demandas del contexto. Por medio de un estudio cualitativo y regional, se busca analizar las experiencias de profesionales de la comunidad educativa de 6 escuelas públicas con Programa de Integración Escolar y en proceso de implementación de la ley de inclusión, en las tres provincias de la Región de Atacama. Con la finalidad de develar realidades latentes a través del análisis de contenido de entrevistas dirigidas a 18 actores educativos: directores, trabajadores sociales y profesionales pertenecientes al Programa de Integración Escolar. En las experiencias y opiniones de quienes participan, se evidencian obstáculos, facilitadores, reticencias y elementos significativos que aportan a la construcción del conocimiento teórico y práctico, principalmente referido a la resistencia a la transformación de la pedagogía tradicional y al trabajo mancomunado con equipos multidisciplinarios, la instalación de prácticas innovadoras mediante adaptaciones curriculares, la importancia del involucramiento de todo el estudiantado y sus familias, la educación desde las emociones y el buen trato, elementos claves para la conformación de comunidades inclusivas.
\end{abstract}

Descriptores: Educación inclusiva; Escuela pública; Política educacional; Profesionales de la educación; Chile.

Chilean education has transitioned in the search of quality and inclusion of everyone, fulfilling the demands of the context. Through a qualitative and local study, experiences from professionals of six educational communities belonging to public schools with an Integration Program in process of implementation due to the inclusion law are analyzed in the three provinces of the Atacama region. The purpose is to reveal unseen realities through the analysis of interviews directed to eighteen educational members: headmasters, social workers and professionals who are part of the Educational Integration Program; the main actors of the current educational process. Through the experiences and arguments of the ones involved in the study; obstacles, enhancements, disagreements, and meaningful elements are made evident. Each one of these help in the scaffolding of theoretical and practical knowledge which mainly refers to the resistance of traditional pedagogical transformation and to the collaborative working developed with multidisciplinary teams, the establishment of innovative practices through curricular adaptions, the importance of students and families' involvement, emotional education and positive behavior, key elements for assembling inclusive communities.

Keywords: Inclusive education; Public school; Education policy; Education professionals; Chile.

*Contacto: adriana.fernandez@uda.cl

ISSN: 2254-3139

www.rinace.net/riejs/

revistas.uam.es/riejs
Recibido: $\quad 29$ de abril 2020

$1^{\text {a }}$ Evaluación: 27 de julio 2020

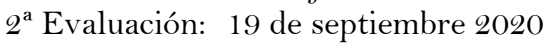

Aceptado: 11 de octubre 2020 


\section{Introducción}

La construcción de una sociedad equitativa, justa e inclusiva es un trabajo complejo que requiere esfuerzos mancomunados para garantizar la igualdad de oportunidades y accesos que satisfagan las necesidades y aseguren el cumplimiento de los derechos fundamentales de los grupos y personas. La educación, en sus distintos modelos y tipos, es un derecho humano fundamental establecido en la Declaración Universal de los Derechos Humanos, ratificada por el Estado de Chile y que propicia el disfrute de los demás derechos, pues desarrolla, entre otros, el principio de igualdad (Castillo-Briceño, 2015).

Hoy, más que nunca, es posible visualizar la heterogeneidad de las personas, familias y colectividades que configuran el escenario social y educativo. La inclusión de la diversidad de niños y niñas, estudiantes y sus necesidades particulares cobra real importancia, pues la segregación y exclusión en esta esfera, limita el desarrollo de las potencialidades individuales y el disfrute pleno de otros derechos. Es por ello, que incansables han sido los esfuerzos por regular y normar el cumplimiento de la garantía inalienable a la educación, sobre todo en los últimos años donde se ha reformulado e innovando en las perspectivas sociales y legislativas en este ámbito.

En este sentido, el objetivo de la presente investigación es "Analizar las experiencias de profesionales de la comunidad educativa en escuelas públicas, con Programa de Integración Escolar, en proceso de implementación de la ley de inclusión, en las tres provincias de la Región de Atacama”, a fin de aportar al desafío de construir investigaciones en el ámbito educativo desde el área local y descentralizada, considerando el contex to social, geográfico y legislativo, a través de los relatos de quienes participan en este proceso: directoras, trabajadores sociales y profesionales del Programa de Integración Escolar (PIE), considerando la concepción de educación inclusiva, la distancia que existe entre la política y el accionar práctico dentro de cada escuelas consultada. Y de esta forma proponer adecuaciones a las políticas educativas nacionales que aseguren la concreción de una educación inclusiva y de calidad (Murillo y Martínez-Garrido, 2019)

El estudio, presenta un recorrido histórico y legislativo, necesario para reconocer el tránsito experimentado hacia el tratamiento actual de inclusión educativa. Por medio del cual, se proyecta constituir bases teóricas y prácticas, enfatizando en la necesidad de instaurar trabajo multidisciplinario y en redes, donde el equipo de profesionales tiene un rol preponderante en la conformación de un clima laboral y una cultura institucional de cohesión y colaboración, que se traduce en una comunidad educativa inclusiva, respetuosa e integral que garantiza al estudiantado y sus familias educación democrática y justa, basada en la diversidad, equidad e igualdad de oportunidades en el acceso y la permanencia (Sánchez, 2012; Villalobos, 2015).

\section{Tránsito hacia una escuela inclusiva}

Los sucesivos y constantes cambios de paradigma han contribuido a los procesos de integración e inclusión paulatina de quienes conforman grupos históricamente excluidos de la sociedad y han servido para la construcción de conocimientos a nivel de experiencias individuales y colectivas en el área de la inclusión (Palacios, 2008). Es así como, el concepto de discapacidad ha ido construyéndose conforme al contexto sociocultural, desde aspectos biológicos y deficitarios hasta uno más integral. Actualmente, se entiende que las personas en esta situación son quienes poseen "deficiencias físicas, mentales, intelectuales 
o sensoriales a largo plazo que, al interactuar con diversas barreras, puedan impedir su participación plena y efectiva en la sociedad, en igualdad de condiciones con las demás" (Asamblea General de las Naciones Unidas, 2006, p. 4). En el área educativa, Chile ha transitado desde una educación que excluyó a personas en esta situación, a la posterior creación de una modalidad de educación especial como alternativa de formación con enfoque integrador.

Hoy, la adopción del concepto de educación inclusiva, enfatiza en la valoración y adaptación a las diversidades y es el ideal educativo nacional (Parra, 2010). En principio, se inauguran diversas escuelas especiales orientadas a la atención de personas con déficit sensoriales, esencialmente sordomudos y luego déficit de carácter intelectual. A mediados de los años sesenta, se reconoce la relación entre quienes se encuentran en situación de discapacidad y su contexto, promoviendo el concepto de normalización, en el que todos tienen derecho a obtener las posibilidades de acceso y participación comunitaria, modificando el ambiente para lograr la integración. Desde entonces y hasta la década del setenta, las escuelas especiales en Chile transforman su enfoque médico, amplían la cobertura de atención y elaboran planes y programas de estudio que incentivan la creación de escuelas especiales privadas (Godoy, Meza y Salazar, 2004).

En este contexto, el Informe Warnock (1978), basado en la Declaración Universal de los Derechos Humanos, emana postulados referidos a la educación como bien universal que busca entregar conocimiento desde la experiencia, promover la independencia y autonomía de acuerdo con las capacidades individuales, eliminando la clasificación de las minusvalías y el etiquetamiento que recibían las personas con discapacidad. Así se instala el término Necesidades Educativas Especiales (NEE), en el que cada estudiante tiene requerimientos particulares que demandan atenciones y recursos educativos específicos, reconociéndose, además, que los problemas de aprendizaje están relacionados con las características individuales y las capacidades de las escuelas para dar respuesta a ellas, siendo primordial contar con los insumos educativos adecuados (Parra, 2010).

En coherencia con estos postulados, entre los años ochenta y noventa, Chile inicia un proceso de reformulación de su sistema educativo con la dictación de la Ley Orgánica Constitucional de Enseñanza (LOCE) (1990), la cual pretende lograr mayor equidad y calidad, procurando avanzar a sistemas integradores e inclusivos. Sin embargo, la educación especial, sitio hasta ese entonces de estudiantes con dificultades de aprendizaje, no fue incluida, evidenciando con ello, que no forma parte de la oferta educativa del país, al omitir planteamientos que den respuesta al estudiantado diverso en el sistema regular ni especial (Godoy, Meza y Salazar, 2004). Menos aún se indican límites en las prácticas de exclusión y expulsión de determinados estudiantes, principalmente de aquellos que parecen presentar mayores dificultades de aprendizaje o menores resultados académicos (Santa Cruz, 2006).

Sin embargo, como respuesta a la influencia internacional, tendiente a promover la inserción conjunta del alumnado con o sin necesidades especiales, se dicta el Decreto $\mathrm{N}^{\circ} 490$ (1990) que promueve la integración escolar de personas con NEE asociadas a discapacidad en establecimientos comunes (Godoy, Meza y Salazar, 2004). Así, se adopta el enfoque de derechos, que establece "la responsabilidad política, jurídica y ética del Estado para hacer cumplir y generar las condiciones de ejercicio pleno de derechos" (Valverde, 2004, p.3), lo que obliga a las partes a diversificar las formas y eliminar las 
barreras y obstaculizadores para alcanzar la inclusión de todas las personas (Parra y Palacios, 2007).

A nivel internacional, la Conferencia Mundial de Jomtien (1990) fundada en el "derecho a una educación para todos", genera bases en el ideario de la inclusión, la cual ofrece satisfacción a las necesidades básicas de aprendizaje al tiempo que desarrolle el bienestar individual y social de las personas dentro del sistema de educación formal. En este mismo sentido, la Conferencia Mundial sobre Necesidades Educativas Especiales: Acceso y Calidad, reafirma la necesidad de brindar enseñanza a todas las personas con NEE dentro del sistema común de educación. La inclusión, entonces, se establece como principio central para la construcción de una educación universal en la escuela común como medio habitual (Duk y Murillo, 2018).

En la misma línea, Chile se propone alcanzar una transformación en su sistema educativo transitando desde un enfoque integrador a uno inclusivo, orientado a la diversidad, realizando esfuerzos estatales, en el ámbito legislativo y financiero, destinado a la circulación hacia la educación regular de estudiantes con discapacidades (Ramos, 2013; Tenorio, 2011). Muestra de ello, es la norma de integración social de las personas con discapacidad en establecimientos educativos (Ley $\mathrm{N}^{\circ}$ 19.284).

Aun así, la concepción clínica del estudiantado con NEE se mantiene, direccionando la intervención hacia la persona, bajo la perspectiva que ésta debe adaptarse al entorno y responsabiliza exclusivamente al equipo de profesionales durante el proceso educativo, lo cual dista del planteamiento original referido a que, la intervención debe ser realizada a todo el contexto, haciendo del estudiantado un agente activo (Hernández, 2015; Ramos, 2013). Pese a la falta de articulación entre las prácticas cotidianas y el ideal legislativo, la integración escolar se convierte en una estrategia clave para la formación de estudiantes en la educación regular (Ramos, 2013).

Contrario a esta propuesta, la Política Nacional de Educación Especial (2005) focaliza los esfuerzos estatales hacia escuelas especiales y no hacia las tradicionales (Ramos, 2013; Tenorio, 2011), provocando dos posturas de pensamiento; por un lado, los estudiantes con discapacidad requieren una educación segregada que les permita compensar los déficits de su situación. Por otro, que un currículo común adaptado con apoyos adicionales puede satisfacer las NEE, permitiendo la inclusión como parte esencial de todo proceso educativo (Godoy, Meza y Salazar, 2004). Desde este último planteamiento, surgen propuestas teóricas, vinculadas al modelo social de discapacidad, el que plantea que todo el alumnado debe tener las mismas oportunidades de acceso, permanencia y desarrollo en las escuelas y en el caso de las NEE, la educación especial sería el último recurso, porque sigue lógicas segregacionistas y dificulta la existencia de un sistema educativo inclusivo e integral; además señala que todo el estudiantado puede presentar algún tipo de NEE y no solo aquellos que tengan discapacidad, es por esto que cada escuela debería adecuarse a sus estudiantes y no al revés (Palacios, 2008, 2017).

Esto se concreta a través de la dictación de la Ley General de Educación, LGE, (2009), la cual provoca un profundo cambio, fortaleciendo la educación pública mediante principios como gratuidad, calidad, equidad, diversidad e integración. Desaparece con ello, la discriminación por particularidades y la diversidad se posiciona como protagonista, para entregar las oportunidades de aprendizaje a cada estudiante, principalmente hacia estudiantes con NEE, al promover las condiciones necesarias para su acceso y retención (López., et al., 2018). En efecto, el Decreto $\mathrm{N}^{\circ} 170$ (2009), establece al Programa de 
Integración Escolar (PIE), como una estrategia de educación inclusiva con miras al logro de objetivos de participación, desarrollo individual y colectivo del estudiantado, principalmente de quienes presentan NEE, ya sean de carácter permanentes o transitorias, al interior del aula común. Para ello, se disponen recursos adicionales y especializados, se fijan las competencias de profesionales docentes y especialistas, con tal de enriquecer sus prácticas docentes y el proceso de evaluación de ingreso del estudiantado y de esta forma, lograr la valoración y respeto a las diferencias y la diversidad (MINEDUC, 2013).

En este contexto nace la Ley de Igualdad de Oportunidades para Personas con Discapacidad (2010), la cual en términos educativos busca asegurar el acceso a las personas con discapacidad a un sistema escolar inclusivo y con igualdad de oportunidades, contempla adecuaciones curriculares, infraestructura y sistema de monitoreo, todo orientado a mejorar los estándares de calidad.

En consecuencia, se aprueba la Ley de Inclusión Escolar (2015) con la finalidad de construir una educación pensada para que todos y todas, independiente de sus diferencias, puedan desarrollar al máximo sus habilidades personales, académicas y sociales (Mesa Técnica Educación Especial, 2015). Fortalece los principios de LGE, enfatizando en la construcción de una educación pública de calidad, orientada a la aceptación de la diversidad, mediante acciones como la eliminación del lucro y aumento de aporte estatal tendiente a la gratuidad universal; sistema de admisión inclusivo, mayor compromiso y apoyo de las familias, sin selección de estudiantes (Valenzuela y Cortese, 2017; López, et al., 2018). Dentro de esta ley, se encuentra el Decreto $N^{\circ} 83$ (2015).

La nueva normativa entrega al PIE un enfoque inclusivo, que implica un profundo cambio en la mirada, transitando desde un paradigma individualista y deficitario, hacia otro que incluye la identificación y eliminación de las barreras del contexto escolar, para educar a la multiplicidad de estudiantes, buscando dar cierre a la dicotomía de perspectivas todavía vigente. Se constituye, entonces, como una señal que permite avanzar hacia una mejor educación a través de la innovación metodológica y diversificación de estrategias para dar cobertura curricular, el trabajo colaborativo y la co-enseñanza (Rodríguez y Ossa, 2014). Elementos que buscan contribuir a la tan anhelada inclusión educativa en todo el territorio nacional.

\section{Caracterización de la realidad educativa en Atacama}

La región de Atacama se ubica al norte de Chile y tiene una superficie de $75.176 \mathrm{~km} 2$, representando el 9,94\% de la superficie del país. Su población alcanza los 286.168 habitantes, con una densidad de 3,81 hab/ km2, siendo urbana en un $91 \%$ (Instituto Nacional de Estadísticas, 2018). Cuenta con una división administrativa de tres provincias y nueve comunas; la capital regional es Copiapó.

Cuadro 1. División territorial administrativa

\begin{tabular}{ll}
\hline \multicolumn{1}{c}{ ProviNCIA } & \multicolumn{1}{c}{ CoMUNAS } \\
\hline Copiapó & Copiapó, Caldera, Tierra amarilla. \\
Chañaral & Chañaral, Diego de Almagro. \\
Huasco & Vallenar, Alto del Carmen, Freirina, Huasco. \\
\hline
\end{tabular}

Fuente: Biblioteca del Congreso Nacional de Chile (2020). 
La última Encuesta de Caracterización Socioeconómica Nacional (CASEN) realizada en el año 2017 , revela que en la región existe un 7,9\% personas pobres por ingreso y un $23,2 \%$ en pobreza multidimensional, dos puntos porcentuales sobre la media nacional. Esta última contempla la medición en base a indicadores de salud, educación, trabajo y seguridad social, vivienda y entorno, redes y cohesión social (Ministerio de Desarrollo Social y Familia, 2018).

Al focalizar el análisis en el indicador educativo, en Chile, el promedio de escolaridad de jefes de hogar de las familias en situación de pobreza multidimensional alcanza los 7,8 años. Además, al desagregar la realidad educacional regional de la población mayor de 18 años, se reconoce que un $13,4 \%$ tiene nula educación formal o enseñanza básica incompleta. A esto se suma, un $13,6 \%$ con educación media incompleta. En tanto, se evidencia una tasa de analfabetismo que alcanza al 3,2\%, frente al 4,3\% a nivel país. Estas cifras evidencian que la educación es un elemento crítico para comprender la pobreza multidimensional en Atacama (Ministerio de Desarrollo Social y Familia, 2018).

Actualmente, la red escolar regional tiene una matrícula de 26.168 estudiantes, distribuidos en 79 establecimientos a lo largo de sus 9 comunas. De ellos, 63\% está ubicado en zonas urbanas y el $86 \%$ corresponde a administración municipal o a Servicios Locales de Educación Pública (Ministerio de Desarrollo Social y Familia, 2018). Todos presentan un Índice de Vulnerabilidad (IVE) superior al 80\%, existiendo 20.055 estudiantes con primera prioridad, lo que significa que el $77 \%$ del total de la matrícula atacameña concentra altas deficiencias en aspectos socioeconómicos, los cuales representan un desafío en el acceso, permanencia y progresión en el sistema educativo formal. La mayor concentración de casos está en la Provincia de Copiapó (Junta Nacional de Auxilio Escolar y becas, 2020).

En este contexto regional se inserta el trabajo con estudiantes con NEE, el que mantiene la tendencia nacional referida a un alto número de establecimientos públicos en el que se insertan. En efecto, durante el año 2018 en Atacama, 87 escuelas regulares implementaron PIE, de las cuales sólo 7 son particulares subvencionadas, el resto corresponde a municipales (MINEDUC, 2019). Según estos antecedentes, es posible distinguir que, en la región, así como en Chile, las escuelas que se reconocen inclusivas, ya sea mediante la declaración de un sello en su Proyecto Educativo o aquellas que solo cuentan con la implementación de PIE, son en su mayoría dependientes del ámbito público, lo cual restringe la inserción de niños y niñas con requerimientos especiales a otros establecimientos, al no estar obligados de ejecutar la normativa vigente.

Las alternativas de elección y acceso al sistema educativo de las que disponen estos estudiantes y sus familias son acotadas y se constituyen como una de las primeras formas de exclusión social, que se traduce en la reproducción de patrones de pobreza multidimensional y desigualdad, situación que se complejiza aún más con el escenario regional. Si bien el Estado ha realizado esfuerzos constantes y significativos por alcanzar la inclusión educativa, estos no se han articulado de forma coherente, por lo que se originan diversos obstáculos, problemáticas y desafíos a la hora de implementar una educación justa y equitativa, con participación activa del estudiantado y los profesionales que componen una comunidad educativa inclusiva, tendiente a fomentar el desarrollo integral, proteger a los grupos vulnerables y garantizar el cumplimiento de los derechos de niños y niñas (Varela, San Martín y Villalobos, 2015). 


\section{Método}

Investigación local y situada cuyo objetivo es analizar las experiencias de profesionales de la comunidad educativa de escuelas públicas con Programa de Integración Escolar, en proceso de implementación de la ley de inclusión, en las tres provincias de la Región de Atacama, con una perspectiva cualitativa, en la que se ahonda en las prácticas, discursos, opiniones e interrelaciones de las personas, desde sus propias ópticas y en relación constante con el ambiente natural donde se desenvuelven. Entonces, fundamental es la consideración de las características constructivistas, dinámicas e integrales de este tipo de estudios, elementos esenciales para su concreción (Rodríguez, Gil y García, 1999).

Desde este enfoque, el procedimiento de muestreo fue configurado como no probabilístico, intencional y de caso-tipo. La muestra estuvo conformada por profesionales de seis escuelas, dos de cada capital provincial de la región de Atacama: Copiapó, Vallenar y Chañaral. Con base en los siguientes criterios de inclusión: escuela de educación básica con administración pública con PIE implementado desde, al menos, un año y dispongan de un/a profesional del trabajo social en su comunidad educativa jornada completa.

En tanto, los participantes de cada establecimiento correspondieron a:

- La persona a cargo de la dirección, cuyo rol lidera y organiza el cumplimiento de su Proyecto Educativo Institucional (PEI), conformando una red de apoyo escolar de impacto transversal en los aprendizajes de todos, sin excepción (MINEDUC, 2013).

- Profesionales especialistas pertenecientes al PIE, que trabajan dentro del aula en co-enseñanza respondiendo a las necesidades del alumnado y se constituyen como agentes de cambio para una cultura y práctica inclusiva tendientes a adoptar estrategias, didácticas y formas de enseñanzas diversas (MINEDUC, 2016).

- Profesionales del trabajo social que ejercen un rol significativo, cuyo quehacer está en directa relación en conformar contextos inclusivos y colaborar en mejorar los procesos y las decisiones pedagógicas (Salazar, 2017). Su labor se vincula con la intervención de estudiantes en conjunto con sus familias y comunidades, procurando reconocer y utilizar sus propios recursos (Castillo y Rodríguez, 2016).

Cuadro 2. Síntesis participantes e instrumentos de recolección

\begin{tabular}{|c|c|c|c|}
\hline CoMUnA & ESCUELAS POR COMUNA & PARTICIPANTES & INSTRUMENTOS \\
\hline Copiapó & \multirow[b]{2}{*}{$\begin{array}{l}6 \text { escuelas básicas con } \\
\text { administración pública }\end{array}$} & \multirow{2}{*}{$\begin{array}{l}1 \text { directora, } \\
1 \text { profesional PIE, } \\
1 \text { trabajador social }\end{array}$} & \multirow{2}{*}{$\begin{array}{l}18 \text { entrevistas } \\
\text { semiestructuradas por } \\
\text { comuna }\end{array}$} \\
\hline Chañaral & & & \\
\hline
\end{tabular}

Fuente: Elaboración propia.

La inserción en el campo implicó la selección de las escuelas de la región según criterios de inclusión y la extensión de invitación a participar. Tras recibir la confirmación por parte de los establecimientos interesados, se realizó el traslado a las capitales provinciales para la ejecución de las entrevistas, teniendo en cuenta la firma de un consentimiento informado por cada participante. 
La recolección de los datos cualitativos se realizó mediante la ejecución de 18 entrevistas semiestructuradas e implicó un proceso simultáneo de transcripción, codificación y análisis de estos, los que fueron tratados mediante análisis de contenido, consistente en la interpretación de las ideas vislumbradas en la conceptualización individual más que de las palabras como tal, sin ignorar al contexto y la relación entre quien investiga y quien es investigado (González-Teruel, 2015). Para asegurar el rigor metodológico del estudio los criterios de confirmabilidad, a través del registro riguroso del proceso investigativo y la credibilidad, por medio de la triangulación de los datos recolectados, estuvieron presentes durante todas las etapas del trabajo de campo.

\section{Resultados}

\subsection{Concepciones de la educación inclusiva}

Quienes participan coinciden, desde una significancia personal, en el entendimiento de educación inclusiva como la apertura y acogida para la entrega de oportunidades equitativas a todo el alumnado, independiente de sus características personales o contextuales. Comprenden también, que es el medio el que debe adaptarse al estudiante y no al revés, pese a que éste no siempre brinda las oportunidades o los recursos para alcanzar este ideal, es por eso que los equipos de trabajo señalan que elementos como la voluntad y vinculación afectiva con la comunidad educativa son claves para construir un entorno inclusivo.

La vinculación afectiva, desde la empatía y los sentimientos, con el estudiantado es primordial para contribuir a la instauración de comunidades educativas seguras, protectoras e inclusivas. (Directora, escuela de Chañaral)

Así mismo, prevalece la idea de que la educación formal e informal son esenciales para alcanzar una sociedad más justa, es por eso que concentran sus esfuerzos en configurar escenarios que propicien la participación y la inclusión en la cotidianeidad escolar, desde aquellas prácticas estrictamente académicas hasta elementos netamente afectivos, emocionales, personales y/o familiares.

De igual modo y desde la significancia profesional, destaca la importancia de educar desde y para las emociones y afectos, considerando los contextos socio-educativos altamente vulnerables en el que están inmersos, cuya realidad es la insuficiencia de recursos requeridos para conformar una comunidad educativa inclusiva o para cumplir con los estándares establecidos en la legislación vigente.

$\mathrm{Al}$ conjugar el sentir personal con el quehacer profesional, se reconocen aspectos símiles, donde las diversidades y las NEE dentro de los establecimientos se dan en todo orden, requiriendo ajustes en lo pedagógico y curricular, pero también en lo relativo a lo conductual, sociocultural y contextual. Así, surge con fuerza el ideario de que la educación inclusiva es un concepto amplio, que debe estar tácita e intrínsecamente contenido en el quehacer de quienes componen las comunidades educativas.

La educación inclusiva es no cuestionárselo, es que no sea tema en tu comunidad educativa, porque cuando no es tema, significa que ya es una escuela inclusiva. (Directora, escuela de Vallenar)

En este sentido, relevando las gestiones más allá del componente académico, destaca el rol del profesional del área social, donde la activación y el seguimiento de redes de colaboración institucional y familiar se articulan con la vinculación afectiva y el 
compromiso personal como elementos claves que enfatizan los discursos de todos quienes participan del estudio y que son ejes fundamentales del trabajador o trabajadora social en estos contextos.

\subsection{El nuevo paradigma del contexto educativo}

Los relatos de los equipos participantes evidencian la voluntad, a nivel profesional, personal e institucional, de constituirse como una comunidad educativa inclusiva, que valora las diversidades en el estudiantado y no discrimina por origen, contexto familiar, nivel socioeconómico, tipos de aprendizaje u otras características individuales. En este sentido, distintas son las adecuaciones y técnicas que han implementado las escuelas, en función de sus recursos, necesidades y capacidad innovadora para ajustarse y responder de la mejor forma a las realidades heterogéneas que enfrentan.

En lo particular y a modo de ejemplo, una escuela de Vallenar indica la instalación de protocolos para el trabajo en inclusión, transversal a toda la comunidad educativa, los cuales se generan a través de consultas y mediciones de satisfacción efectuadas a estudiantes y sus familias cada año, entendiendo la necesidad de participación de todos quienes conforman las comunidades educativas y el mejoramiento continuo en su proceder. Por otro lado, una escuela de Chañaral con sello inclusivo, revela la importancia del buen trato en las relaciones dentro del establecimiento, que además de mejorar la calidad educativa, impacta positivamente en el reconocimiento de la comunidad y resultados de pruebas estandarizadas. Por tanto, la vinculación afectiva y la valoración de las diferencias son esenciales para la optimización de las escuelas y sus procesos.

Los recursos limitados con los que disponen los establecimientos, son en general, una de las grandes dificultades para dar respuesta al estudiantado diverso, a los estándares legales y, en definitiva, para la consecución de una comunidad realmente inclusiva en cuanto a lo académico, infraestructura, formación y capacitación constante, principalmente. En cuanto a esto, todas las escuelas reconocen que la complementariedad de los programas SEP (Subvención Escolar Preferencial) y PIE, ofrece oportunidades en la utilización y reasignación de tales recursos como práctica cotidiana para sortear algunos requerimientos; además de la capacitación mutua constante y el trabajo colaborativo que se da entre los equipos profesionales de ambos programas.

\section{En la parte que estamos al debe es en el recurso y muchos recursos lo hemos solucionado en gran parte con la SEP, pero no es todo, es mucho, más mucho mucho más allá. (Directora, escuela de Vallenar)}

Aun así, los equipos directivos, principalmente, reconocen la importancia de las reformas educativas y encabezan los trabajos para adecuarse a ellas, se encargan de motivar y supervisar la gestión de los equipos, en los cuales se brinda gran importancia a elementos como la voluntad y la disposición de las personas al cambio y al trabajo mancomunado. Considerando que actualmente, no solo la emergente nueva legislación les exhorta al cambio de paradigma, también lo hacen los contextos socioculturales diversos, complejos y con altos índices de vulnerabilidad a los que se ven enfrentados las escuelas y sus profesionales.

$\Upsilon$ en ese sentido, los directivos estamos comprometidos con generar nuevos enfoques, generar nuevas posibilidades, no solamente para los estudiantes, sino que también para nuestros docentes y asistentes. (Directora, escuela de Chañaral) 


\subsection{Formación permanente}

En las escuelas consultadas, la formación y capacitación continua es un elemento muy valorado por los participantes, la cual se da a través de los equipos PIE, mediante talleres acordes al mínimo exigido por ley y relacionados al tratamiento pedagógico de casos específicos. Estas instancias se reconocen como pertinentes al llevarse a cabo en el mismo contexto educacional y por profesionales expertos en las temáticas y en la realidad local. Su realización ha permitido, además del abordaje de las diversidades, posicionar la temática de la inclusión y disminuir la resistencia al trabajo con estudiantes con NEE.

\section{La capacitación es realizada por PIE, lo cual es valorado positivo por realizarla en base al mismo contexto y con la misma gente de la comunidad. (Trabajador Social, escuela de Vallenar)}

Pese a la existencia de tales instancias de formación, estas no son suficientes ni satisfacen todas las necesidades de conocimiento para dar respuesta a los requerimientos cotidianos. Sin embargo, los entrevistados señalan que se ejecutan, de forma excepcional, talleres extraordinarios para dar cumplimiento a contingencias, como, por ejemplo, el surgimiento de una NEE no abordada previamente por la comunidad educativa. Destaca en una escuela de Vallenar y una de Chañaral, la inversión en recursos adicionales, extraídos del Plan de Mejoramiento Educativo para capacitar a través de organismos externos a la escuela, las que son bien recibidas por los equipos de trabajo.

Surge también, en los relatos de los entrevistados, el hecho de que pese a los esfuerzos realizados por los equipos directivos, la aplicación de los conocimientos adquiridos en cuanto a prácticas y metodologías diversas, depende de cada docente. La inclusión, desde esta perspectiva, queda entonces relegada a las voluntades, al no siempre existir un sistema de monitoreo al desarrollo de cada clase o medición de resultados esperados.

\subsection{Inclusión y práctica educativa}

Las personas entrevistadas que no cumplen roles pedagógicos, reconocen como elemento común en los docentes con mayor experiencia laboral, el tránsito experimentado desde una férrea oposición en la adopción de estrategias diversas basado principalmente en la resistencia a la instalación de la lógica inclusiva que obliga a atender en forma diferenciada al estudiantado, además de la entrada de otros profesionales al aula, hasta llegar a la flexibilización e integración de éstas en su cotidianeidad. Gracias, principalmente, a las exigencias de las reformas legales, las instancias de formación y la visualización de los buenos resultados que se han obtenido. De esta forma, la educación inclusiva se ha ido construyendo de forma paulatina y constante.

Hubo una resistencia bastante grande ¿ya? y esta cosa del poco conocimiento que ellos
expresaban tener para trabajar con estos niños con necesidades educativas especiales,
pero se comenzó con esta ley, se comenzó como a capacitar, comenzó como muy lento,
(...) Y esto comenzó como a crecer, porque los profesores se empezaron a dar cuenta de
que el trabajo de adecuaciones curriculares empezó a ser importante y que si se
requerían para aquellos alumnos que estaban dentro de la sala(...) no se podían dejar
solos, sin hacer nada o dibujando o que hicieran alguna otra situación, sino que había
que dedicar tiempo, recursos y también un poco de estrategias diversas para poder
atenderles. (Coordinadora PIE, escuela de Copiapó)

En este escenario, resalta la estrategia similar declarada por una escuela de Copiapó y una de Vallenar, consistente en adelantar procesos asociados al abordaje de NEE, previo a la obligación legal, con el fin de dar mayor tiempo y holgura a la adaptación, obteniendo así el cumplimiento de la norma y resultados satisfactorios. 
Actualmente y sin perder de vista la lógica de tránsito descrita, los agentes educativos se refieren a estrategias pedagógicas, adaptaciones curriculares o Diseño Universal para el Aprendizaje (DUA) y co-docencia como las más reconocidas, concretas y efectivas tanto para estudiantes con NEE como para el grupo curso en general. Transversalmente se declara que estas instancias son desarrolladas en las horas asignadas por carga laboral de docentes de lenguaje y matemáticas en coordinación con profesionales PIE.

Si bien, en general, las experiencias de las comunidades educativas atacameñas son similares entre sí, surgen diferencias significativas que tienen que ver con la gestión, el enfoque y el sello que cada escuela le da a su perspectiva de inclusión. En este sentido, es importante indicar que, en una escuela de Vallenar, se realiza trabajo con docentes de otros subsectores, según demanda espontánea, ampliando la cobertura de las estrategias mencionadas. Por otra parte, en Chañaral, una escuela declara contar con apoyo de un profesional curriculista, quien participa en la construcción del DUA y otras adecuaciones necesarias.

Se demuestra así, la concreción de uno de los componentes esenciales del PIE, la coenseñanza dentro del aula entre profesionales especialistas y el profesorado regular de asignaturas. Potenciando con ello, el trabajo colaborativo y la adecuación constante a los nuevos desafíos que impone la realidad escolar particular, como el sistema educativo en general.

En complemento con el quehacer pedagógico y en atención al abordaje integral de la realidad del estudiantado, los y las trabajadoras sociales entrevistados, coinciden en que pese a lo fundamental de su labor en la atención al proceso socio-familiar que incide en el aprendizaje, existen todavía una amplia resistencia por parte de los docentes para permitir su ingreso al aula y establecer coordinación permanente. Constituyéndose como una limitante para la ejecución de planes de intervención y el abordaje holístico de cada realidad, instaurando una perspectiva segregadora que impide el aprovechamiento óptimo de todos los recursos y redes disponibles de cada estudiante y su contexto.

En este sentido, destaca el rol de la trabajadora social en una escuela de Chañaral, cuyo elemento distintivo es el trabajo en redes establecido con la escuela especial en el proceso de traslado de sus estudiantes al establecimiento de enseñanza regular. Esta articulación ha permitido entregar los apoyos y soportes necesarios en atención a la adaptación del estudiante a la nueva realidad educativa, impidiendo una posible deserción o retroceso en su progresión académica y a la vez, un monitoreo de la capacidad de respuesta del equipo profesional a los requerimientos emergentes.

\subsection{En cuanto al equipo multidisciplinario PIE}

Respecto al funcionamiento de los equipos PIE de las escuelas de Atacama, los participantes refieren que existe una gran demanda, por lo que deben ampliar constantemente la cobertura de las prestaciones del programa, desarrollando la dinámica del "sobrecupo", en la que se atiende a más estudiantes con NEE de los estipulados en la normativa, apelando a la optimización de los recursos escasos con los que disponen y, principalmente, a la voluntad de los profesionales del equipo, quienes además coinciden en que existe una sobrecarga en las gestiones administrativas asociadas a su rol, por lo que es complejo compatibilizar con el quehacer educativo.

En el mismo ámbito, los profesionales relatan que los diagnósticos biomédicos priman a la hora de determinar el ingreso al PIE, relegando factores sociales, familiares y 
contextuales que igualmente constituyen necesidades educativas especiales y requieren de distintos tipos de apoyos que podrían brindarse desde el programa. Estos elementos conjugados, dificultan su funcionamiento y se constituyen en desafíos diarios para dar respuesta a las distintas realidades del estudiantado.

Las directoras participantes, destacan el rol y el quehacer fundamental de los equipos multidisciplinarios que se involucran en los procesos de construcción de comunidades educativas inclusivas, ya sea que estos profesionales se vinculen directamente o no con el funcionamiento del PIE, desempeñan una labor primordial en el desarrollo de estrategias y abordaje integral de las diversidades.

A pesar de la importancia de la perspectiva multidisciplinaria en el tratamiento de la diversidad educativa y la alta valoración del quehacer del trabajo social con estudiantes PIE y sus familias, considerando sus necesidades multidimensionales y los contextos comunitarios de las escuelas públicas, no se cuenta con profesionales del trabajo social adscritos o que trabajen directamente con el equipo PIE. Solo una escuela de Chañaral declara la asignación de 6 horas semanales a la trabajadora social para labores relacionadas con este programa.

Una trabajadora social en terreno, haciendo visita domiciliaria, o sea, ha sido de apoyo para nosotros, de bastante de apoyo. $\Upsilon$ cómo ellos están organizados, tienen bastante unión como grupo, como equipo y se involucran en toda la actividad de la escuela. (Directora, escuela de Chañaral)

El trabajo multidisciplinario, entonces, es tangible principalmente a través de los espacios de capacitación, reflexión y/o colaboración, tendientes a la organización y planificación de la atención hacia el estudiantado diverso. Dichas instancias, están programadas o son convocadas de forma habitual por los profesionales del programa y son muy bien acogidas por los agentes educativos. No obstante, queda en evidencia en los relatos recogidos, que la relación entre el equipo multidisciplinario y el profesorado regular ha sido compleja, principalmente por la paulatina y resistida instalación de una perspectiva inclusiva, que insta a atender las particularidades de cada estudiante, implementando estrategias innovadoras a través del trabajo conjunto con otros profesionales mediante la co-docencia, lo que implica la entrada de otros participantes al aula en una lógica de coordinación permanente y por tanto, convierte la forma tradicional de hacer docencia.

Con el paso del tiempo, las gestiones directivas y la visualización de resultados, este proceso se constituye como un desafío que se enfrenta comprendiendo a la inclusión como una construcción que requiere esfuerzos mancomunados y voluntades para generar transformaciones significativas en el quehacer académico, el clima organizacional y en el trabajo colaborativo.

\subsection{Las prácticas inclusivas y sus resultados}

Las prácticas inclusivas, como la implementación de estrategias pedagógicas diversas y el ideario de la equidad en el trato y las oportunidades, propician un clima escolar favorable para toda la comunidad educativa, impactando en distintos ámbitos, más allá de los estándares de medición académico, que si bien es importante para quienes participan de la investigación, no es un elemento fundamental, pues centran sus discursos en los logros y resultados cualitativos, destacando el aspecto relacional, la colaboración entre estudiantes y la percepción de sí mismos como pares. Resultados que se obtienen tras el trabajo coordinado y constante y se constituyen como un impulso y motivación que fortalece a las comunidades educativas y enaltece su trabajo. 
Aun así, una escuela en Chañaral y otra en Vallenar, declaran también contar con productos y resultados cuantitativos concretos de sus prácticas inclusivas, como la mejora en resultados de pruebas nacionales estandarizadas, disminución en la tasa de deserción escolar, alta satisfacción de las familias en encuestas auto-aplicadas, inserción y permanencia de estudiantes PIE en la enseñanza media con rendimiento favorable, conducente inclusive al egreso, lo que evidentemente es un logro importante y re-significa la labor que realizan.

En cambio, las experiencias son poco alentadoras a la hora de vincular a las familias con los agentes educativos, ya que en todas las escuelas se reconoce que no existe una relación o vínculo funcional ni estrecho entre ellos, lo que se constituye como un gran obstáculo. Pese a ello, es común en los discursos de los profesionales, la necesidad imperiosa de participación activa y constante de parte de los grupos familiares en los procesos de sus estudiantes, sobre todo si estos requieren de adecuaciones curriculares u otros apoyos. La familia es parte primordial de la comunidad educativa y debe hacerse participe para que las prácticas inclusivas y los esfuerzos de los equipos profesionales sean significativos y efectivos.

Cuesta lograr el compromiso de los apoderados, que asistan, que vengan a los talleres que uno quiere hacer, que entienda que a los niños los tienen que mandar regularmente, porque si tienen problemas de aprendizaje y no los mandan a la escuela es más difícil que aprendan. (Trabajadora Social, escuela Chañaral)

\section{Discusión}

Las experiencias de los y las profesionales de las comunidades educativas de las escuelas públicas con Programa de Integración Escolar, en proceso de implementación de la ley de inclusión, en las tres provincias de la Región de Atacama, develan que la inclusión es un concepto amplio, que debe estar tácita e intrínsecamente contenido en el quehacer de quienes son parte de los establecimientos. Es un proceso vigente e inspirador que está en desarrollo y en el que se enaltecen las diversidades, en cuanto a perfil del estudiante, contextos de origen, caracterización familiar u otros elementos diferenciadores, bajo el reconocimiento de que la educación es un derecho que debe ser brindado en igualdad de condiciones (Duk y Murillo, 2018; Palacios, 2017).

También, revelan que la interacción social, la voluntariedad, el trabajo colaborativo, la educación basada en las emociones y el buen trato por parte de quienes participan en los procesos educativos inclusivos, son fundamentales para alcanzar el ideal legislativo actual (Rojas, Falabella y Alarcón, 2016). Los entrevistados valoran en términos profesionales y personales las diversidades en las aulas, indican que enriquecen a la comunidad y tiene resultados favorables tanto para quienes presentan necesidades educativas especiales como para aquellos que forman parte del alumnado regular.

Tal escenario surge principalmente por la cobertura total actual, la cual concentra en el aula a distintas culturas, capital económico, habilidades y necesidades, siendo la escuela un espacio privilegiado para la inclusión social y la formación en valores que acoja y aprecie la diversidad, considerando que es en este espacio, donde co-habitan individuos con intereses, motivaciones y capacidades diferentes (Lacuesta y Traver-Martí, 2018). Aun así, todavía se evidencia la utilización de un lenguaje poco inclusivo que deja entrever características despectivas hacia el estudiantado, señal inequívoca de que la inclusión es 
un proceso que está todavía en construcción (Mena, Muñoz y Cortese, 2013; Valenzuela y Cortese, 2017).

Los constantes cambios sociopolíticos instalan nuevas demandas que obligan a implementar múltiples e innovadores esfuerzos efectuados por cada escuela en respuesta a las diversas demandas de sus estudiantes (Vila, González y Martín, 2020). Pese a ello, es transversal la insuficiencia de recursos, lo cual merma las posibilidades de concreción de una comunidad escolar capaz de acoger todas las particularidades (Lledó y Arnaiz, 2010). Esto puede comprenderse al reconocer que el sistema educativo chileno cuenta con una administración centralizada y estandarizada que ha tardado en identificar las necesidades y características locales en los distintos contextos regionales que distan de ser similares entre sí.

En este escenario, el PIE se constituye como eje fundamental de la implementación de estrategias innovadoras que buscan que el aprendizaje propiciado hacia un grupo heterogéneo se transforme en un proceso educativo que responda a necesidades particulares por medio de la adecuación curricular, trabajo colaborativo, diversificación curricular y la co-enseñanza como estrategias de educación inclusiva; acciones que se ciñen a lo que la ley indica (Rodríguez y Ossa, 2014), comprendiendo que el ajuste al ritmo de cada uno, facilita el proceso de aprendizaje más que sobrecarga el quehacer académico en el aula.

Asimismo, genera espacios de formación docente para afrontar el trabajo con estudiantes con dificultades de aprendizaje, permitiendo con ello que los establecimientos tengan acceso a profesionales calificados que respondan a las necesidades de forma oportuna. Este tratamiento ha implicado transitar hacia el fin de la lógica de resistencia profesional e iniciar un proceso de aceptación al abordaje metodológico colaborativo mediante la codocencia y la formación permanente. Sin embargo, la falta de continuidad de estos espacios de capacitación se reconoce como una debilidad (Duk, Cisternas y Ramos, 2019). A lo que se suma, la escasa supervisión hacia la efectiva aplicación de los nuevos aprendizajes, quedando relegada su utilización a la voluntad de cada docente.

Dentro del funcionamiento interno del programa, se aprecia además de elementos de sobrecarga administrativa, una exclusiva integración de estudiantes bajo un diagnóstico biomédico, ignorando elementos sociales y emocionales (Cobeñas, 2020). Además de la normalización del trabajo con alumnos por sobre la cobertura asignada, apelando a la voluntad y vinculación socio-afectiva de los profesionales, para la reasignación de recursos y la entrega de atención oportuna sobre todo a aquellos que presentan mayores dificultades o se constituyen como más vulnerables. Factores que obligan a la realización de adecuaciones que traspasan el actuar dentro del aula y permea a todos los actores educativos (Cobeñas, 2020).

En este ámbito, la Ley de Subvención Escolar Preferencial (SEP) favorece la inclusión al entregar recursos adicionales a estudiantes vulnerables, con el fin de garantizar la equidad de oportunidades y la educación como un valor público (López et al., 2018); pero en las escuelas consultadas ésta se constituye como la única alternativa de financiamiento para emprender acciones complementarias al PIE, en el mejoramiento de las condiciones de educación que brindan al estudiantado.

En este contexto, las escuelas se constituyen como agentes protectores dentro de la sociedad, por lo que en su interior se debe velar por la inclusión, generando estrategias 
diversas dentro y fuera del aula para que exista equidad en acceso, participación y permanencia del estudiantado.

Además, la necesidad de educar con un componente afectivo, desde y para las emociones, se configura como un imperativo en los profesionales consultados, toda vez que se reconoce allí la clave para la conformación de comunidades verdaderamente inclusivas. De esta forma, el equilibrio emocional de los niños y adolescentes con NEE transitorias o permanentes, requiere obtener del hogar el necesario apoyo emocional de los hijos y de la escuela el desarrollo de estrategias didácticas específicas orientadas en la misma dirección (Alegre y Villar, 2019). En directa relación con este propósito, el rol de los y las trabajadoras sociales tiende a la mejora constante de los procesos y las decisiones que experimentan las escuelas (Salazar, 2017), sus intervenciones se vinculan con el entorno en el que el estudiantado se desenvuelve, teniendo una perspectiva amplia y compleja de la realidad, estableciendo un nexo entre "la cultura escolar y las formas culturales" presentes en el estudiantado, todo lo cual otorga un aporte significativo a la labor de los equipos interdisciplinarios (Castillo y Rodríguez, 2016; MINEDUC, 2013). En la mejora educativa, convivencia y derecho a la educación, sobre todo de los más vulnerables, donde comúnmente se posicionan los estudiantes con NEE.

Sin embargo, pese a la relevancia de sus funciones, el trabajo social se ve desconectado del sistema de medición de prácticas inclusivas y de la progresión académica del estudiante, debido a variadas limitantes, entre ellas aquellas relacionadas con asignación de horas para el trabajo complementario con equipo PIE y profesorado regular. Además del insuficiente involucramiento de los equipos educativos en la realidad sociofamiliar del estudiantado, restando importancia e impacto a la intervención integral que un trabajador social realiza con el estudiante, sus redes familiares, sociales y comunitarias.

Dentro de los desafíos diarios, surgen limitaciones en recursos económicos y físicos, además del requerimiento constante de actualización y formación profesional acorde los nuevos y cambiantes requerimientos del alumnado (Lledó y Arnaiz, 2010). A su vez, el traspaso a la administración estatal mediante la figura de Servicios Locales de Educación Pública, genera una nueva incertidumbre en el funcionamiento de las escuelas, apreciándose como un proceso administrativo, burocrático y descontextualizado de los requerimientos de cada región, comuna o escuela.

A esto se suma la falta de involucramiento por parte de las familias en los procesos educativos, situación que podría comprenderse al reconocer el perfil educativo de los jefes de hogar atacameños, quienes poseen bajo nivel de instrucción y presentan una elevada pobreza multidimensional, transversalizando las vulnerabilidades e intensificando las desigualdades en los contextos socio familiares del alumnado (Cobeñas, 2020). Elementos que conjugan un escenario donde la relevancia por la educación y el cumplimiento del rol de soporte al aprendizaje, pierde fuerza y merma el impacto de las acciones de inclusión (Ministerio de Desarrollo Social, 2017). En este sentido, se reconoce que las familias constituyen una pieza clave en la construcción de las comunidades educativas, su participación activa en este proceso potencia el trabajo del equipo profesional, lo hace relevante y facilita la proyección del estudiantado y la consecución de mejores resultados tanto para las escuelas como para los niños y niñas (Simón, Giné y Echeita, 2016; Vila, Cortés y Martín, 2020).

En definitiva, los antecedentes expuestos evidencian que la disposición y compromiso de las personas que trabajan dentro de estos contextos altamente demandantes y versátiles 
es fundamental para generar instancias inclusivas y climas organizacionales saludables. Labores coherentes y coordinadas entre el profesorado, dirección, administración y quienes les asisten, se traducen en el logro de una educación integral, inclusiva y de calidad (Cobeñas, 2020; MINEDUC, 2016). Hoy las escuelas se proyectan respetando el sentido de la ley de inclusión escolar, la cual promueve la construcción de una educación de calidad con orientaciones claras hacia la aceptación de la diversidad, mediante la eliminación del lucro y aumento de aporte estatal tendiente a la gratuidad universal; sistema de admisión inclusivo, con mayor compromiso y apoyo de las familias, sin selección de estudiantes (Valenzuela y Cortese, 2017).

Pese a ello, la conformación de comunidades educativas inclusivas en las escuelas de Atacama, avanza a paso lento, acorde a los ideales de los equipos directivos, atendiendo principalmente a la adecuación de estándares para dar cumplimiento a la normativa vigente, siempre basados en elementos de voluntariedad y compromiso de los actores pertenecientes a cada comunidad educativa.

\section{Referencias}

Alegre, O. y Villar L. (2019). Relación entre los problemas emocionales y el desarrollo del lenguaje en niños y adolescentes con dificultades auditivas. REICE. Revista Iberoamericana sobre Calidad, Eficacia y Cambio en Educación, 17(1), 5-23. https://doi.org/10.15366/reice2019.17.1.001

Asamblea General de las Naciones Unidas. (2006). Convención sobre los derechos de las personas con discapacidad y su protocolo facultativo. Asamblea General de las Naciones Unidas.

Biblioteca del Congreso Nacional de Chile. (2020). División política-administrativa, Chile nuestro país, Región de Atacama. Biblioteca del Congreso Nacional de Chile.

Castillo, V. y Rodríguez, C. (2016). Los aportes de los asistentes de la educación: La escuela como campo de intervención para el trabajo social. Revista Cuaderno de Trabajo Social, 8(1), 62-84.

Castillo-Briceño, C. (2015). Posicionando la educación inclusiva: Una forma diferente de mirar el horizonte educativo. Revista Educación, 39(2), 123-152.

https://doi.org/10.15517/revedu.v39i2.19902

Cobeñas, P. (2020). Exclusión educativa de personas con discapacidad: Un problema pedagógico. REICE. Revista Iberoamericana sobre Calidad, Eficacia y Cambio en Educación, 18(1), 65-81. https://doi.org/10.15366/reice2020.18.1.004

Duk, C. y Murillo, F. J. (2018). El mensaje de la educación inclusiva es simple, pero su puesta en práctica es compleja. Revista Latinoamericana de Educación Inclusiva, 12(1), 11-13. https://doi.org/10.4067/S0718-73782018000100011

Duk, C., Cisternas, T. y Ramos, L. (2019). Formación docente desde un enfoque inclusivo. A 25 años de la declaración de salamanca, nuevos y viejos desafíos. Revista Latinoamericana de Educación Inclusiva, 13(2), 91-109. https://doi.org/10.4067/S07 18-73782019000200091

Godoy, M., Meza, M. y Salazar A. (2004). Antecedentes históricos, presente y futuro de la educación especial en Chile. MINEDUC.

González-Teruel, A. (2015). Estrategias metodológicas para la investigación del usuario en los medios sociales: Análisis de contenido, teoría fundamentada y análisis del discurso. El Profesional de la Información, 24(3), 32 1-328. https://doi.org/10.3145/epi.2015.may.12 
Hernández, M. (2015). El concepto de discapacidad: De la enfermedad al enfoque de derechos. Revista CES Derecho, 6(2), 46-59.

Instituto Nacional de Estadísticas. (2018). Síntesis resultados. Censo 2017. INE.

Junta Nacional de Auxilio Escolar y Becas. (2020). Índice de vulnerabilidad escolar. JUNAEB

Lacuesta, D. y Traver-Martí, J. A. (2018). Yo, contigo. El programa de mediación entre iguales, desde la perspectiva de un ejemplo de vida. REICE. Revista Iberoamericana sobre Calidad, Eficacia y Cambio en Educación, 16(1), 53-71. https://doi.org/10.15366/reice2018.16.1.004

Lledó, A. y Arnaiz, P. (2010). Evaluación de las prácticas educativas del profesorado de los centros escolares: Indicadores de mejora desde la educación inclusiva. REICE. Revista Iberoamericana sobre Calidad, Eficacia y Cambio en Educación, 8(5), 96-109.

http://www.rinace.net/reice/numeros/arts/vol8num5/art6.pdf

López, V., González, P., Manghi, D., Ascorra, P., Oyanedel, J. C., Redón, S., Leal, F. y Salgado, M. (2018). Políticas de inclusión educativa en Chile: Tres nudos críticos. Educational Policy and Analysis Archives, 26(157), 1-24. https://doi.org/10.14507/epaa.26.3088

Ministerio de Desarrollo Social y Familia. (2018). CASEN 2017: Encuesta de caracterización socioeconómica nacional. http://observatorio.ministeriodesarrollosocial.gob.cl/casenmultidimensional/casen/casen_2017.php

MINEDUC. (2013). Orientaciones técnicas para programas de integración escolar PIE. MINEDUC.

MINEDUC. (2016). Manual de apoyo a la inclusión escolar en el marco de la reforma educacional. MINEDUC.

MINEDUC. (2019). Estadísticas de la educación 2018. MINEDUC.

Mena, I., Muñoz, B. y Cortese, I. (2012). El desafío de la diversidad en el sistema escolar. En I. Mena, M. Lissi, L. Alcalay y N. Milicic (Eds.), Educación y diversidad: Aportes desde la psicología educacional (pp.19-44). Ediciones UC.

Murillo, F. J. y Martínez-Garrido, C. (2019). Una mirada a la investigación educativa en América Latina a partir de sus artículos. REICE. Revista Iberoamericana sobre Calidad, Eficacia y Cambio en Educación, 17(2), 5-25. https://doi.org/10.15366/reice2019.17.2.001

Palacios, A. (2008) El modelo social de discapacidad: Orígenes, caracterización y plasmación en la Convención Internacional sobre los Derechos de las Personas con Discapacidad. Ediciones CINCA.

Palacios, A. (2017). El modelo social de discapacidad y su concepción como cuestión de derechos humanos. Revista Colombiana de Ciencias Sociales, 8(1), 14-18. http://doi.org/10.21501/22161201.2190

Parra, C. (2010). Educación inclusiva: Un modelo de educación para todos. Revista ISEES, 8, 7384. https://doi.org/10.22518/16578953.767

Parra, C. y Palacios, M. (2007). Enfoque de derechos humanos en la política pública de discapacidad. Civilizar. Ciencias Sociales y Humanas, 2, 97-114.

Ramos, L. (2013). Educación especial y educación inclusiva en Chile: ¿En punto de estancamiento? Revista Latinoamericana de Inclusión Educativa, 7(2), 37-46.

Rodríguez, F. y Ossa, C. (2014). Valoración del trabajo colaborativo entre profesores de escuelas básicas de Tomé, Chile. Estudios Pedagógicos, 40(2), 303-319.

https://doi.org/10.4067/SO7 18-07052014000300018

Rodríguez, G., Gil, J. y García, E. (1999). Metodología de la investigación cualitativa. Aljibe. 
Rojas, M., Falabella, A. y Alarcón, P. (2016). Inclusión social en las escuelas: Estudio de prácticas pedagógicas inclusivas y proyecciones para enfrentar un escenario sin copago y selección escolar. FONIDE.

Salazar, A. (2017). Avances en la diversificación de la enseñanza. MINEDUC.

Sánchez, N. (2012). El currículo de la educación básica en México: Un proyecto educativo flexible para la atención a la diversidad y el fortalecimiento de la sociedad democrática. REICE. Revista Iberoamericana sobre Calidad, Eficacia y Cambio en Educación, 10(4), 149-163. http://www.rinace.net/reice/numeros/arts/vol10num4/art10.pdf

Santa Cruz, E. (2006). Sobre la LOCE y el escenario actual. Revista Docencia, 29, 17-29.

Simón, C., Giné, C. y Echeita, G. (2016). Escuela, familia y comunidad: Construyendo alianzas para promover la inclusión. Revista Latinoamericana de Inclusión Educativa, 1O(1), 25-42. https://doi.org/10.4067/S0718-73782016000100003

Valenzuela, C. y Cortese, I. (2017). Transitando hacia una educación inclusiva: Breve revisión a los paradigmas y leyes. Valoras UC.

Valverde, F. (2004). Apuntes sobre enfoque de derechos. ACHNU.

Varela, C., San Martín, C. y Villalobos, C. (2015). Opciones educativas para alumnos que presentan NEE en el sistema educativo actual: ¿Coherencia con una reforma hacia la educación inclusiva? Informes para la Política Educativa, 9, 1-9.

Vila, E., Cortés, P. y Martín, V. (2020). Los educadores y educadoras sociales en los centros educativos de Andalucía: Perfil y desarrollo profesional. REICE. Revista Iberoamericana sobre Calidad, Eficacia y Cambio en Educación, 18(1), 47-64. https://doi.org/10.15366/reice2020.18.1.003

Villalobos, C. (2015). El rol de la intervención social en el sistema escolar chileno. Revista Intervención Universidad Alberto Hurtado, 4, 56-62.

Tenorio, S. (2011). Formación inicial docente y necesidades educativas especiales. Estudios Pedagógicos, 37(2), 249-265. http://doi.org/10.4067/S0718-07052011000200015.

\section{Breve CV de las autoras}

\section{Adriana Fernández Muñoz}

Académica en Dpto. de Trabajo Social de la Universidad de Atacama, Copiapó. Trabajadora Social, Magíster en Trabajo Social y Políticas Sociales. Diplomada en Investigación y Pedagogía Universitaria, Gobierno y Gestión Pública y Docencia Universitaria. Ha participado en investigaciones en líneas de exclusión social y vulnerabilidades; formación general en Trabajo Social e inclusión educativa. ORCID ID: https://orcid.org/OOOO-OOO1-6562-3514.Email: adriana.fernandez@uda.cl

\section{Daniela Durán Rojas}

Académica en Dpto. de Trabajo Social de la Universidad de Atacama, Copiapó. Trabajadora Social, Magíster en Metodologías de Investigación Cualitativa para la Salud. Diplomada en Liderazgo Social. Ha participado en líneas de investigación relativas a la inclusión educativa, discapacidades y género. ORCID ID: https://orcid.org/oOOO-OOO21626-8154. Email: daniela.duran@uda.cl 